\title{
UPPER BOUNDS FOR SUNFLOWER-FREE SETS
}

\author{
ERIC NASLUND ${ }^{1}$ and WILL SAWIN ${ }^{2}$ \\ ${ }^{1}$ Mathematics Department, Princeton University, Fine Hall, Washington Road, Princeton, \\ NJ 08544, USA; \\ email: naslund@princeton.edu \\ ${ }^{2}$ ETH Institute for Theoretical Studies, ETH Zurich, 8092 Zürich, Switzerland; \\ email: william.sawin@math.ethz.ch
}

Received 16 July 2016; accepted 3 November 2016

\begin{abstract}
A collection of $k$ sets is said to form a $k$-sunflower, or $\Delta$-system, if the intersection of any two sets from the collection is the same, and we call a family of sets $\mathcal{F}$ sunflower-free if it contains no 3-sunflowers. Following the recent breakthrough of Ellenberg and Gijswijt ('On large subsets of $\mathbb{F}_{q}^{n}$ with no three-term arithmetic progression', Ann. of Math. (2) 185 (2017), 339-343); ('Progression-free sets in $\mathbb{Z}_{4}^{n}$ are exponentially small', Ann. of Math. (2) 185 (2017), 331-337) we apply the polynomial method directly to Erdős-Szemerédi sunflower problem (Erdős and Szemerédi, 'Combinatorial properties of systems of sets', J. Combin. Theory Ser. A 24 (1978), 308-313) and prove that any sunflower-free family $\mathcal{F}$ of subsets of $\{1,2, \ldots, n\}$ has size at most

$$
|\mathcal{F}| \leqslant 3 n \sum_{k \leqslant n / 3}\left(\begin{array}{l}
n \\
k
\end{array}\right) \leqslant\left(\frac{3}{2^{2 / 3}}\right)^{n(1+o(1))} .
$$

We say that a set $A \subset(\mathbb{Z} / D \mathbb{Z})^{n}=\{1,2, \ldots, D\}^{n}$ for $D>2$ is sunflower-free if for every distinct triple $x, y, z \in A$ there exists a coordinate $i$ where exactly two of $x_{i}, y_{i}, z_{i}$ are equal. Using a version of the polynomial method with characters $\chi: \mathbb{Z} / D \mathbb{Z} \rightarrow \mathbb{C}$ instead of polynomials, we show that any sunflower-free set $A \subset(\mathbb{Z} / D \mathbb{Z})^{n}$ has size

$$
|A| \leqslant c_{D}^{n}
$$

where $c_{D}=\frac{3}{2^{2 / 3}}(D-1)^{2 / 3}$. This can be seen as making further progress on a possible approach to proving the Erdôs and Rado sunflower conjecture ('Intersection theorems for systems of sets', J. Lond. Math. Soc. (2) 35 (1960), 85-90), which by the work of Alon et al. ('On sunflowers and matrix multiplication', Comput. Complexity 22 (2013), 219-243; Theorem 2.6) is equivalent to proving that $c_{D} \leqslant C$ for some constant $C$ independent of $D$.

2010 Mathematics Subject Classification: 05D05 (primary); 05B40 and 11B30 (secondary)

(c) The Author(s) 2017. This is an Open Access article, distributed under the terms of the Creative Commons Attribution licence (http://creativecommons.org/licenses/by/4.0/), which permits unrestricted re-use, distribution, and reproduction in any medium, provided the original work is properly cited.
\end{abstract}




\section{Introduction}

A collection of $k$ sets is said to form a $k$-sunflower, or $\Delta$-system, if the intersection of any two sets from the collection is the same. A family of sets $\mathcal{F}$ is said to be $k$-sunflower-free if no $k$ members form a $k$-sunflower, and when $k=3$ we simply say that the collection $\mathcal{F}$ is sunflower-free. It is a longstanding conjecture that sunflower-free families must be small, and there are two natural situations in which we may ask this question. The first, and most general case, is when each set in the family has size $m$. Erdős and Rado made the following conjecture which is now known as the Sunflower Conjecture.

CONJECTURE 1 (Erdős-Rado Sunflower Conjecture [5]). Let $k \geqslant 3$, and suppose that $\mathcal{F}$ is a $k$-sunflower-free family of sets, each of size $m$. Then

$$
|\mathcal{F}| \leqslant C_{k}^{m}
$$

for a constant $C_{k}>0$ depending only on $k$.

In their paper, Erdôs and Rado [5] proved that any $k$-sunflower-free family of sets of size $m$ has size at most $m !(k-1)^{m}$, but the conjectured bound of $C_{k}^{m}$ remains out of reach for any $k \geqslant 3$. The second setting for upper bounds for $k$-sunflower-free sets concerns the case where each member of $\mathcal{F}$ is a subset of the same $n$-element set. There can be at most $2^{n}$ such subsets, and the Erdős-Szemerédi sunflower conjecture states that this trivial upper bound can be improved by an exponential factor.

Conjecture 2 (Erdős-Szemerédi sunflower conjecture [6]). Let $S$ be a $k$ sunflower-free collection of subsets of $\{1,2, \ldots, n\}$. Then

$$
|S|<c_{k}^{n}
$$

for some constant $c_{k}<2$ depending only on $k$.

In Erdôs and Szemerédi's paper [6], they prove that Conjecture 2 follows from Conjecture 1 (see also [1, Theorem 2.3]), and so it is a weaker variant of the sunflower problem. Let $F_{k}(n)$ denote the size of the largest $k$-sunflower-free collection $\mathcal{F}$ of subsets of $\{1,2, \ldots, n\}$, and define

$$
\mu_{k}^{S}=\limsup _{n \rightarrow \infty} F_{k}(n)^{1 / n}
$$

to be the Erdös-Szmerédi-k-sunflower-free capacity. The trivial bound is $\mu_{k}^{S} \leqslant 2$, and the Erdős-Szemerédi sunflower conjecture states that $\mu_{k}^{S}<2$ for all $k \geqslant 3$. 
In this paper we prove new bounds for the sunflower-free capacity $\mu_{3}^{S}$. It is a theorem of Alon et al. [1, page 7] that the recent work of Ellenberg and Gijswijt [4] on progression-free sets in $\mathbb{F}_{3}^{n}$ implies that $\mu_{3}^{S}<2$, and before this, the best upper bound for a sunflower-free collection of $\{1,2, \ldots, n\}$ was $2^{n} \exp (-c \sqrt{n})$ due to Erdős and Szemerédi [6]. We give a simple proof of a quantitative version of [1, page 7], showing that $\mu_{3}^{S} \leqslant \sqrt{1+C}$ where $C$ is the capset capacity. However, using the ideas from the recent breakthrough of Ellenberg and Gijswijt and Croot et al. $[3,4]$ on progressions in $\mathbb{F}_{3}^{n}$, and from Tao's version of the argument [8], we apply the polynomial method directly to this problem, and obtain a stronger result:

THEOREM 1. Let $\mathcal{F}$ be a sunflower-free collection of subsets of $\{1,2, \ldots, n\}$. Then

$$
|\mathcal{F}| \leqslant 3(n+1) \sum_{k \leqslant n / 3}\left(\begin{array}{l}
n \\
k
\end{array}\right),
$$

and

$$
\mu_{3}^{S} \leqslant \frac{3}{2^{2 / 3}}=1.889881574 \cdots
$$

There is still a large gap between upper and lower bounds for the sunflower-free capacity $\mu_{3}^{S}$.

In Section 3 we turn to the sunflower problem in the set $\{1,2, \ldots, D\}$, which we always think of as $\mathbb{Z} / D \mathbb{Z}$. Alon, Shpilka and Umans [1, Definition 2.5], defined a $k$-sunflower in $(\mathbb{Z} / D \mathbb{Z})^{n}$ for $k \leqslant D$ to be a collection of $k$ vectors such that in each coordinate they are either all different or all the same. When $k=3$ and $D=3$ this condition is equivalent to being a three-term arithmetic progression in $\mathbb{F}_{3}^{n}$.

CONJECTURe 3 (Sunflower conjecture in $(\mathbb{Z} / D \mathbb{Z})$ ). Let $k \leqslant D$, and let $A \subset(\mathbb{Z} / D \mathbb{Z})^{n}$ be a $k$-sunflower-free set. Then

$$
|A| \leqslant b_{k}^{n}
$$

for a constant $b_{k}$ depending only on $k$.

The motivation for this problem comes from [1, Theorem 2.6] where they proved that Conjecture 3 is equivalent to the Erdős-Rado sunflower conjecture. In particular, if there exists a constant $b_{3}$ independent of $D$ such that any 3 -sunflowerfree set in $(\mathbb{Z} / D \mathbb{Z})^{n}$ has size at most $b_{3}^{n}$, then Conjecture 1 holds for $k=3$ with $c_{3}=e \cdot b_{3}$. Since a sunflower-free set cannot contain a three-term arithmetic progression, the recent result of Ellenberg and Gijswijt [4] implies an upper bound 
for sunflower-free sets $A \subset(\mathbb{Z} / D \mathbb{Z})^{n}$ for $D$ prime of the form $|A| \leqslant c_{D}^{n}$, where $c_{D}=D e^{-I((D-1) / 3)}$ for a function $I$ defined in [4] in terms of a certain optimization problem. It is not too hard to see that

$$
0<\lim _{D \rightarrow \infty} \frac{c_{D}}{D}<1 .
$$

Using the characters $\chi: \mathbb{Z} / D \mathbb{Z} \rightarrow \mathbb{C}$ instead of polynomials, we prove the following theorem:

THEOREM 2. Let $D \geqslant 3$, and let $A \subset(\mathbb{Z} / D \mathbb{Z})^{n}$ be a sunflower-free set. Then

$$
|A| \leqslant c_{D}^{n}
$$

where $c_{D}=\frac{3}{2^{2 / 3}}(D-1)^{2 / 3}$.

This can be seen as progress towards the Erdôs-Rado sunflower conjecture, and we remark that the now resolved Erdős-Szemerédi conjecture for $k=3$ is equivalent to proving that $c_{D}<D^{1-\epsilon}$ for some $\epsilon$ and all $D$ sufficiently large [1, Theorem 2.7].

To prove Theorems 1 and 2 we bound the slice rank of a function of three variables $T(x, y, z)$ which is nonvanishing if and only if $x=y=z$ or $x, y, z$ form a sunflower.

A function $f: A^{k} \rightarrow \mathbb{F}$, where $A^{k}=A \times A \times \cdots \times A$ denotes the Cartesian product and $\mathbb{F}$ is a field, is said to be a slice if it can be written in the form

$$
f\left(x_{1}, \ldots, x_{k}\right)=h\left(x_{i}\right) g\left(x_{1}, \ldots, x_{i-1}, x_{i+1}, \ldots, x_{k}\right)
$$

where $h: A \rightarrow \mathbb{F}$ and $g: A^{k-1} \rightarrow \mathbb{F}$. The slice rank of a general function $f:$ $A^{k} \rightarrow \mathbb{F}$ is the smallest number $m$ such that $f$ is a linear combination of $m$ slices. If $A$ is a sunflower-free set, it follows that, for $x, y, z \in A, T(x, y, z)$ is nonzero if and only if $x=y=z$.

We then have the following lemma:

LEMMA 1 (Rank of diagonal hypermatrices [8, Lemma 1]). Let $A$ be a finite set and $\mathbb{F}$ a field. Let $T(x, y, z)$ be a function $A \times A \times A \rightarrow \mathbb{F}$ such that $T(x, y, z) \neq 0$ if and only if $x=y=z$. Then the slice rank of $T$ is equal to $|A|$.

Using this lemma, we need only find an upper bound on the slice rank of $T$ to obtain an upper bound on the size of the sunflower-free set. In each case we do this by an explicit decomposition of $T$ into slices found by writing $T$ as either a polynomial or as a sum of characters. We refer the reader to [2, Section 4] for further discussion of the slice rank. 
This method is the direct analogue of Tao's interpretation [8] of the EllenbergGijswijt argument for capsets, and can be thought of as a 3-tensor generalization of the Haemer bound [7] which bounds the Sperner capacity of a hypergraph rather than the Shannon capacity of a graph.

We stress two differences between our result and several other papers which use the slice rank method $[2,8]$, or which have been reinterpreted to use the slice rank $[3,4]$. First, these papers study functions valued in finite fields, whose characteristic is chosen for the specific problem and cannot be changed without affecting the bound. Our work uses functions valued in a field of characteristic zero, though we could have done the same thing in any finite field of sufficiently large characteristic. Second, these papers mainly describe functions as low-degree polynomials and use that structure to bound their slice rank. In the proof of Theorem 2, we describe functions as sums of characters. One can interpret characters as polynomials restricted to the set of roots of unity, but under this interpretation the degree of the polynomial is not relevant to the proof of Theorem 2 - only the number of nontrivial characters is.

REMARK 1. The proofs of Theorems 1 and 2 can be extended without modification to handle a multicoloured version of the problem analogous to multicoloured sum-free sets as defined in [2].

\section{The Erdôs-Szemerédi sunflower problem}

Any subset of $\{1,2, \ldots, n\}$ corresponds to a vector in $\{0,1\}^{n}$ where a 1 or 0 in coordinate $i$ denotes whether or not $i$ lies in the subset. A sunflower-free collection of subsets of $\{1,2, \ldots, n\}$ gives rise to a set $S \subset\{0,1\}^{n}$ with the property that for any three distinct vectors $x, y, z \in S$, there exists $i$ such that $\left\{x_{i}, y_{i}, z_{i}\right\}=\{0,1,1\}$.

Moreover, a sunflower-free collection of subsets of $\{1,2, \ldots, n\}$ that also does not contain two subsets with one a proper subset of the other gives rise to a set $S \subset\{0,1\}^{n}$ such that for any $x, y, z \in S$ not all equal, there exists $i$ such that $\left\{x_{i}\right.$, $\left.y_{i}, z_{i}\right\}=\{0,1,1\}$. This holds because the only new case is when two are equal and the third is not (say $x=y$ and $z$ is distinct), and then because $x \neq z, x$ is not a subset of $z$, so there exists some $i$ such that $x_{i}=y_{i}=1$ and $z_{i}=0$.

Given a sunflower-free set $S \subset\{0,1\}^{n}$, let $S_{l}$, for $l=1, \ldots, n$, denote the elements of $S$ with exactly $l$ ones so that $S=\bigcup_{l=0}^{n} S_{l}$. Then for each $l, S_{l}$ is a sunflower-free collection of subsets with none a proper subset of another, hence whenever $x, y, z \in S_{l}$ satisfy $x+y+z \notin\{0,1,3\}^{n}$ we must have $x=y=z$. For $x, y, z \in\{0,1\}^{n}$ consider the function $T:\{0,1\}^{n} \times\{0,1\}^{n} \times\{0,1\}^{n} \rightarrow \mathbb{R}$ given 
by

$$
T(x, y, z)=\prod_{i=1}^{n}\left(2-\left(x_{i}+y_{i}+z_{i}\right)\right) .
$$

The function $T(x, y, z)$ is nonvanishing precisely on triples $x, y, z$ such that there does not exist $i$ where $\left\{x_{i}, y_{i}, z_{i}\right\}=\{1,1,0\}$. Hence restricted to $S_{l} \times S_{l} \times S_{l}, T(x$, $y, z)$ is nonzero if and only if $x=y=z$. So by Lemma 1 , the slice rank of $T$ is at least $\left|S_{l}\right|$. Expanding the product form for $T(x, y, z)$, we may write $T(x, y, z)$ as a linear combination of products of three monomials

$$
x_{1}^{i_{1}} \cdots x_{n}^{i_{n}} y_{1}^{j_{1}} \cdots y_{n}^{j_{n}} z_{1}^{k_{1}} \cdots z_{n}^{k_{n}}
$$

where $i_{1}, \ldots, i_{n}, j_{1}, \ldots, j_{n}, k_{1}, \ldots, k_{n} \in\{0,1\}^{n}$, and

$$
i_{1}+\cdots+i_{n}+j_{1}+\cdots+j_{n}+k_{1}+\cdots+k_{n} \leqslant n .
$$

For each product of three monomials, at least one of $i_{1}+\cdots+i_{n}, j_{1}+\cdots+j_{n}$, $k_{1}+\cdots+k_{n}$ is at most $n / 3$. For each term in $T$, choose either $x_{1}^{i_{1}} \cdots x_{n}^{i_{n}}, y_{1}^{j_{1}} \cdots y_{n}^{j_{n}}$, or $z_{1}^{k_{1}} \cdots z_{n}^{k_{n}}$, making sure to choose one of total degree at most $n / 3$. Divide the expansion of $T$ into, for each possible monomial, the sum of all the terms where we chose that monomial. Because one monomial in each of these sums is fixed, we can express each sum as a product of that monomial (a function of one variable) times the sum of all the other terms (a function of the other variables), hence each of these sums is a slice. The total slice rank is at most the number of slices, which is at most the number of monomials we can choose: three times the number of monomials in $n$ variables of degree at most 1 in each variable and of total degree at most $k$. The number of such monomials is exactly $\sum_{k \leqslant n / 3}\left(\begin{array}{l}n \\ k\end{array}\right)$, so this yields the upper bound

$$
\begin{gathered}
\left|S_{l}\right| \leqslant 3 \sum_{k \leqslant n / 3}\left(\begin{array}{l}
n \\
k
\end{array}\right), \\
|S| \leqslant \sum_{l=0}^{n}\left|S_{l}\right| \leqslant 3(n+1) \sum_{k \leqslant n / 3}\left(\begin{array}{l}
n \\
k
\end{array}\right)
\end{gathered}
$$

which is the statement of Theorem 1 .

2.1. Capset capacity. A capset $A$ is a subset of $\mathbb{F}_{3}^{n}$ containing no three-term arithmetic progressions. Let $A_{n} \subset \mathbb{F}_{3}^{n}$ denote the largest capset in dimension $n$, and define

$$
C=\limsup _{n \rightarrow \infty}\left|A_{n}\right|^{1 / n}
$$


to be the capset capacity. Note that $\left|A_{n}\right|$ is super-multiplicative, that is for $m$, $n \geqslant 1$ we have $\left|A_{m n}\right| \geqslant\left|A_{n}\right|^{m}$ since $A_{n}$ Cartesian-producted with itself $m$ times is a capset in $\mathbb{F}_{3}^{m n}$. Ellenberg and Gijswijt [4] proved that $C \leqslant 2.7552$, and the following theorem is a quantitative version of a result of Alon et al. [1, page 7].

THEOREM 3. We have that $\mu_{3}^{S} \leqslant \sqrt{1+C}$ where $C$ is the capset capacity and $\mu_{3}^{S}$ is the Erdôs-Szmeredi-sunflower-free capacity.

Proof. We bound the size of the largest sunflower-free set in $\{0,1\}^{2 n}$ by writing each vector in terms of the four vectors in $\{0,1\}^{2}$

$$
u_{0}=\left[\begin{array}{l}
0 \\
0
\end{array}\right], \quad u_{1}=\left[\begin{array}{l}
1 \\
0
\end{array}\right], \quad u_{2}=\left[\begin{array}{l}
0 \\
1
\end{array}\right], \quad u_{3}=\left[\begin{array}{l}
1 \\
1
\end{array}\right] .
$$

Every set $S \subset\{0,1\}^{2 n}$ corresponds to a set $\tilde{S} \in\{0,1,2,3\}^{n}$ where we obtain $S$ from $\tilde{S}$ by replacing each symbol $i$ for $i \in\{0,1,2,3\}$ with the vector $u_{i}$. For example,

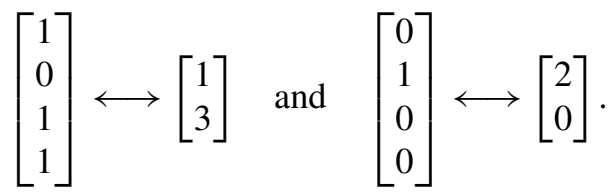

For each $x \in\{0,1\}^{n}$ consider

$$
\tilde{S}_{x}=\left\{v \in \tilde{S}: v_{i}=3 \text { if and only if } x_{i}=1\right\} .
$$

We may view elements of $\tilde{S}_{x}$ as elements of $\{0,1,2\}^{n-x}=\mathbb{F}_{3}^{n-x}$ by ignoring the coordinates where $x$ is 1 . If three elements in $\tilde{S}_{x}$ form an arithmetic progression in $\mathbb{F}_{3}^{n-x}$, then in each coordinate the elements of $\tilde{S}_{x}$ are either all the same or are $0,1,2$ in any order, so the entries of the corresponding vectors in $S$ are either all the same or $u_{0}, u_{1}, u_{2}$ in any order. Because $u_{0}, u_{1}, u_{2}$ form a sunflower, these three elements of $S$ form a sunflower. Because $S$ is a sunflower-free set, $\tilde{S}_{x}$ is a capset. Let $w(x)=\sum_{i=1}^{n} x_{i}$ be the weight of the vector $x$, then

$$
\left|\tilde{S}_{x}\right| \leqslant C^{n-w(x)}
$$

where $C$ is the capset capacity. Hence

$$
|S| \leqslant \sum_{x} C^{n-w(x)}=\sum_{j=0}^{n}\left(\begin{array}{l}
n \\
j
\end{array}\right) C^{n-j}=(1+C)^{n},
$$

and we obtain the desired bound.

Using the Ellenberg-Gijswijt upper bound on capset capacity, this gives $\mu_{3}^{S} \leqslant 1.938$, which is not as strong a bound as Theorem 1 . 


\section{Sunflower-free sets in $(\mathbb{Z} / D \mathbb{Z})^{n}$}

Consider the $D$ characters $\chi: \mathbb{Z} / D \mathbb{Z} \rightarrow \mathbb{C}^{\times}$. By the orthogonality relations, for any $a, b \in \mathbb{Z} / D \mathbb{Z}$

$$
\frac{1}{|D|} \sum_{\chi} \chi(a-b)= \begin{cases}1 & \text { if } a=b \\ 0 & \text { otherwise }\end{cases}
$$

Hence

$$
\begin{aligned}
& \frac{1}{|D|} \sum_{\chi}(\chi(a) \overline{\chi(b)}+\chi(b) \overline{\chi(c)}+\chi(a) \overline{\chi(c)}) \\
& \quad= \begin{cases}0 & \text { if } a, b, c \text { are distinct, } \\
1 & \text { if exactly two of } a, b, c \text { are equal, } \\
3 & \text { if } a=b=c .\end{cases}
\end{aligned}
$$

For $x, y, z \in(\mathbb{Z} / D \mathbb{Z})^{n}$, define the function $T:(\mathbb{Z} / D \mathbb{Z})^{n} \times(\mathbb{Z} / D \mathbb{Z})^{n} \times$ $(\mathbb{Z} / D \mathbb{Z})^{n} \rightarrow \mathbb{C}$ by

$$
\begin{aligned}
& T(x, y, z)=\prod_{j=1}^{n}\left(\frac{1}{|D|} \sum_{\chi}\left(\chi\left(x_{i}\right) \overline{\chi\left(y_{i}\right)}+\chi\left(y_{i}\right) \overline{\chi\left(z_{i}\right)}+\chi\left(x_{i}\right) \overline{\chi\left(z_{i}\right)}\right)-1\right) \\
& =\prod_{j=1}^{n}\left(\frac{1}{|D|} \sum_{\chi}\left(\chi\left(x_{i}\right) \overline{\chi\left(y_{i}\right)} 1\left(z_{i}\right)+1\left(x_{i}\right) \chi\left(y_{i}\right) \overline{\chi\left(z_{i}\right)}+\chi\left(x_{i}\right) 1\left(y_{i}\right) \overline{\chi\left(z_{i}\right)}\right)\right. \\
& \left.\quad-1\left(x_{i}\right) 1\left(y_{i}\right) 1\left(z_{i}\right)\right)
\end{aligned}
$$

which is nonzero if and only if $x, y, z$ form a $\mathbb{Z} / D \mathbb{Z}$-sunflower or are all equal. Let $A \subset(\mathbb{Z} / D \mathbb{Z})^{n}$ be a sunflower-free set. Then restricted to $A \times A \times A, T$ is nonzero if and only if $x=y=z$. Hence by Lemma 1 the slice rank of $T$ is at least $|A|$. Expanding the product in (1), we see that $T$ can be written as a linear combination of terms of the form

$$
\chi_{1}\left(x_{1}\right) \cdots \chi_{n}\left(x_{n}\right) \psi_{1}\left(y_{1}\right) \cdots \psi_{n}\left(y_{n}\right) \xi_{1}\left(z_{1}\right) \cdots \xi_{n}\left(z_{n}\right)
$$

where $\chi_{1}, \ldots, \chi_{n}, \psi_{1}, \ldots, \psi_{n}, \xi_{1}, \ldots, \xi_{n}$ are characters on $\mathbb{Z} / D \mathbb{Z}$, at most $2 n$ of which are nontrivial. For any such term, at least one of the tuples $\chi_{1}, \ldots, \chi_{n}$, $\psi_{1}, \ldots, \psi_{n}, \xi_{1}, \ldots, \xi_{n}$ must contain at most $2 n / 3$ nontrivial characters. Grouping 
the terms by the one containing the fewest nontrivial characters like this, we are able to upper bound the slice rank of $T$ by

$$
3 \sum_{k \leqslant 2 n / 3}\left(\begin{array}{l}
n \\
k
\end{array}\right)(D-1)^{k},
$$

where the $(D-1)^{k}$ comes from the fact that for each set of $k$ indices, we have $(D-1)^{k}$ possible choices of nontrivial characters. Because $D \geqslant 3,(D-1) / 2 \geqslant 1$, so:

$$
\begin{aligned}
\sum_{k \leqslant 2 n / 3}\left(\begin{array}{l}
n \\
k
\end{array}\right)(D-1)^{k} \leqslant \sum_{k \leqslant 2 n / 3}\left(\begin{array}{l}
n \\
k
\end{array}\right)(D-1)^{k}\left(\frac{D-1}{2}\right)^{2 n / 3-k} \\
=\left(\frac{D-1}{2}\right)^{-n / 3} \sum_{k \leqslant 2 n / 3}\left(\begin{array}{l}
n \\
k
\end{array}\right)(D-1)^{k}\left(\frac{D-1}{2}\right)^{n-k} \\
\leqslant\left(\frac{D-1}{2}\right)^{-n / 3} \sum_{k \leqslant n}\left(\begin{array}{l}
n \\
k
\end{array}\right)(D-1)^{k}\left(\frac{D-1}{2}\right)^{n-k} \\
=\left(\frac{D-1}{2}\right)^{-n / 3}\left(D-1+\frac{D-1}{2}\right)^{n}=\left(\frac{3}{2^{2 / 3}}(D-1)^{2 / 3}\right)^{n} .
\end{aligned}
$$

Let $c_{D}=\frac{3}{2^{2 / 3}}(D-1)^{2 / 3}$. This inequality proves that for $|A|$ a sunflower-free set,

$$
|A| \leqslant 3 c_{D}^{n}
$$

To prove Theorem 2 (that $|A| \leqslant c_{D}^{n}$ ), we can remove the factor of 3 by a standard amplification argument, as for $A$ a sunflower-free set in $(\mathbb{Z} / D \mathbb{Z})^{n}, A^{k}$ is a sunflower-free set in $(\mathbb{Z} / D \mathbb{Z})^{n k}$, so $|A| \leqslant\left(3 c_{D}^{n k}\right)^{1 / k}=3^{1 / k} c_{D}^{n}$. Taking $k \rightarrow \infty$, we obtain Theorem 2.

\section{Acknowledgements}

Eric Naslund was supported by Ben Green's ERC Starting Grant 279438, Approximate Algebraic Structure and Applications. William F. Sawin was supported by NSF grant DGE-1148900, Dr. Max Rössler, the Walter Haefner Foundation, and the ETH Zürich Foundation.

\section{References}

[1] N. Alon, A. Shpilka and C. Umans, 'On sunflowers and matrix multiplication', Comput. Complexity 22 (2013), 219-243.

[2] J. Blasiak, T. Church, H. Cohn, J. Grochow, E. Naslund, W. F. Sawin and C. Umans, 'On cap sets and the group-theoretic approach to matrix multiplication', Discrete Anal. 3 (2017). 
[3] E. Croot, V. Lev and P. Pach, 'Progression-free sets in $\mathbb{Z}_{4}^{n}$ are exponentially small', Ann. of Math. (2) 185 (2017), 331-337.

[4] J. S. Ellenberg and D. Gijswijt, 'On large subsets of $\mathbb{F}_{q}^{n}$ with no three-term arithmetic progression', Ann. of Math. (2) 185 (2017), 339-343.

[5] P. Erdős and R. Rado, 'Intersection theorems for systems of sets', J. Lond. Math. Soc. (2) 35 (1960), 85-90.

[6] P. Erdős and E. Szemerédi, 'Combinatorial properties of systems of sets', J. Combin. Theory Ser. A 24 (1978), 308-313.

[7] W. Haemers, 'An upper bound for the Shannon capacity of a graph', in Algebraic Methods in Graph Theory, Vols. I, II (Szeged, 1978), Colloq. Math. Soc. János Bolyai, 25, 267-272.

[8] T. Tao, 'A symmetric formulation of the Croot-Lev-Pach-Ellenberg-Gijswijt capset bound, blog post', 2016, http://terrytao.wordpress.com/2016/05/18/a. 